University of Wollongong

Research Online

Faculty of Engineering and Information

Faculty of Engineering and Information

Sciences - Papers: Part A

Sciences

1996

Far-infrared measurements of cyclotron resonance and impurity transitions in bulk $n-G a A s$ in pulsed magnetic fields

\author{
$\mathrm{R} A$. Lewis \\ University of Wollongong, roger@uow.edu.au \\ R J. Heron \\ University of Wollongong \\ R G. Clark \\ University of New South Wales \\ R P. Starrett \\ University of New South Wales
}

A V. Skougarevsky

University of New South Wales

Follow this and additional works at: https://ro.uow.edu.au/eispapers

Part of the Engineering Commons, and the Science and Technology Studies Commons

Research Online is the open access institutional repository for the University of Wollongong. For further information contact the UOW Library: research-pubs@uow.edu.au 


\title{
Far-infrared measurements of cyclotron resonance and impurity transitions in bulk $n-G a A s$ in pulsed magnetic fields
}

\author{
Abstract \\ The photoconductive response of $n-G a A s$ has been measured under laser radiation in the wavelength \\ range 71 to $513 / \mathrm{spl} \mathrm{mu/m}$ at temperatures as low as $400 \mathrm{mK}$ and at magnetic fields as high as $42 \mathrm{~T}$. A \\ rich variety of spectral features are observed: cyclotron resonance, transitions associated with $D /$ sup $-/$, \\ and transitions to stable and metastable hydrogenic states. The use of a pulsed magnet has extended the \\ magnetic field range over which data is available by a factor of three.

\section{Keywords} \\ measurements, cyclotron, transitions, bulk, far-infrared, fields, n-gaas, magnetic, pulsed, resonance, \\ impurity \\ Disciplines \\ Engineering | Science and Technology Studies

\section{Publication Details} \\ Lewis, R. A., Heron, R. J., Clark, R. G., Starrett, R. P. \& Skougarevsky, A. V. (1996). Far-infrared \\ measurements of cyclotron resonance and impurity transitions in bulk n-GaAs in pulsed magnetic fields. \\ 1996 Conference on Optoelectronic and Microelectronic Materials And Devices: Proceedings (pp. \\ 325-328). United States: IEEE.
}




\title{
Far-infrared measurements of cyclotron resonance and impurity transitions in bulk n-GaAs in pulsed magnetic fields
}

\author{
R. A. Lewis and R. J. Heron \\ Department of Physics, University of Wollongong, Wollongong NSW 2522, Australia \\ R. G. Clark, R. P. Starrett and A. V. Skougarevsky \\ School of Physics, University of New South Wales, Kensington NSW 2033, Australia
}

\begin{abstract}
The photoconductive response of $n$-GaAs has been measured under laser radiation in the wavelength range 71 to $513 \mu \mathrm{m}$ at temperatures as low as $400 \mathrm{mK}$ and at magnetic fields as high as $42 \mathrm{~T}$. A rich variety of spectral features are observed: cyclotron resonance, transitions associated with $\mathrm{D}^{-}$, and transitions to stable and metastable hydrogenic states. The use of a pulsed magnet has extended the magnetic field range over which data is available by a factor of three.
\end{abstract}

\section{A. Introduction}

The conduction band of GaAs is relatively simple. It is almost parabolic and isotropic and the minimum lies at the zone centre. These properties make the application of effective mass theory to $\mathrm{n}$-GaAs very straight-forward. Electrons bound to donor sites form analogues of the hydrogen atom, scaled by virtue of the electron effective mass being $6.65 \%$ of the free electron mass, and the dielectric constant being 12.56 rather than 1 . The binding energy of this hydrogen analogue is thus $\mathrm{E}_{\mathrm{b}}=0.0665 /(12.56)^{2} \times 13.6 \mathrm{eV}=5.72 \mathrm{meV}$ and the Bohr radius is $\mathrm{r}=12.56 / .0665 \times 0.529 \AA=100 \AA$. A second electron may be bound to the donor site producing $\mathrm{D}^{-}$, the solid state analogue of the hydrogen ion $\mathrm{H}^{-}$.

Just as visible and near-visible spectroscopy yields much information about atomic hydrogen, so far-infrared radiation, of wavelength 40 to $1000 \mu \mathrm{m}$, or energy approximately 1 to $30 \mathrm{meV}$, is a potent probe of the solid-state analogues of $\mathrm{H}^{\text {and }} \mathrm{H}^{-}$.

The application of a magnetic field to the hydrogen atom adds structure to the energy states. The conduction band continuum is quantised into Landau levels. The bound states undergo Zeeman splitting. Additional hydrogenic states, which are stable only at infinite magnetic field and have no zero-field atomic counterpart, appear.

In this paper the far-infrared magneto-spectroscopy of bulk n-GaAs is described. Data is given for cyclotron resonance, transitions from $\mathrm{D}^{-}$to the $\mathrm{N}=1$ Landau level, and transitions from the hydrogenic ground state to excited stable and metastable states. The dimensionless quantity used to measure energy is the Rydberg, which has the value of 13.6 $\mathrm{eV}$ for hydrogen and $5.72 \mathrm{meV}$ for the electron in GaAs. The dimensionless quantity used to measure magnetic field is $\gamma=\hbar \omega / 2 \mathrm{Ry} ; \gamma=1$ corresponds to a magnetic field of $6.57 \mathrm{~T}$ in GaAs and to a field of $2.35 \times 10^{5} \mathrm{~T}$ for the hydrogen atom.

\section{B. Experimental methods}

The large magnetic fields employed in this investigation (up to $42 \mathrm{~T}$ ) were generated at the National Pulsed Magnet Laboratory, which is fully described elsewhere [1]. The farinfrared radiation was supplied by an optically pumped laser (Edinburgh Instruments) and conducted through a top-loading probe (Oxford Instruments) into a ${ }^{3} \mathrm{He}$ cryostat. This arrangement, which is described in detail elsewhere [2], allows far-infrared spectroscopy to be carried out at simultaneous high magnetic field (to $50 \mathrm{~T}$ ) and low temperature (to $300 \mathrm{mK}$ ). 


\section{Results and discussion}

\section{C.1 Cyclotron resonance}

Cyclotron resonance was observed as a small peak for most laser wavelengths employed. The peak positions are shown in Fig. 1. The dashed line has a slope of 2 and zero intercept and represents the cyclotron resonance position for a constant effective mass of $0.0665 \mathrm{~m}_{\mathrm{e}}$. At higher magnetic field the data falls slightly below this line (i.e. the effective mass increases slightly with field), but the conduction band is parabolic to a good approximation. Lower-temperature data $(300 \mathrm{mK})$ is found to be in good agreement this data and with previous results reported at $4.2 \mathrm{~K}$.

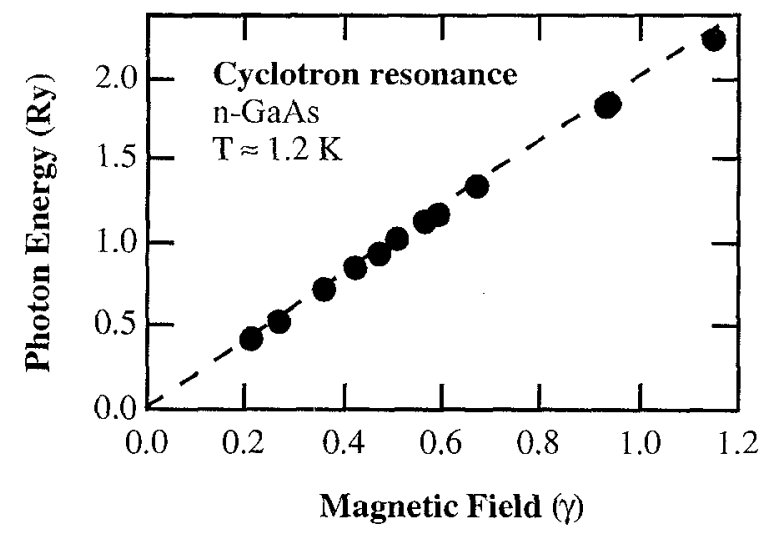

Fig. 1: Cyclotron resonance in $\mathrm{n}$-GaAs. $\mathrm{Ry}=5.72 \mathrm{meV}$ and $\gamma=6.57 \mathrm{~T}$. The dashed line shows $\mathrm{m}^{*} / \mathrm{m}_{\mathrm{e}}=0.0665$.

\section{C.2 $D^{-}$transitions}

Observed at lower magnetic field than the cyclotron resonance for a given laser line, not extrapolating back to zero energy at zero field, and present at lower energies than any of the hydrogenic transitions, are a set of transitions ascribed to $\mathrm{D}^{-}$[3]. The mechanism of detection is excitation directly into a Landau level. The data in Fig. 2 correspond to excitation to $\mathrm{N}$ $=1$. Differentiating the spectra has allowed transitions up to $\mathrm{N}=6$ to be detected, but no feature corresponding to $\mathrm{N}=0$ has been observed. Our data in Fig. 2, taken at lower temperature, is in good agreement with that given in the earlier work [3].

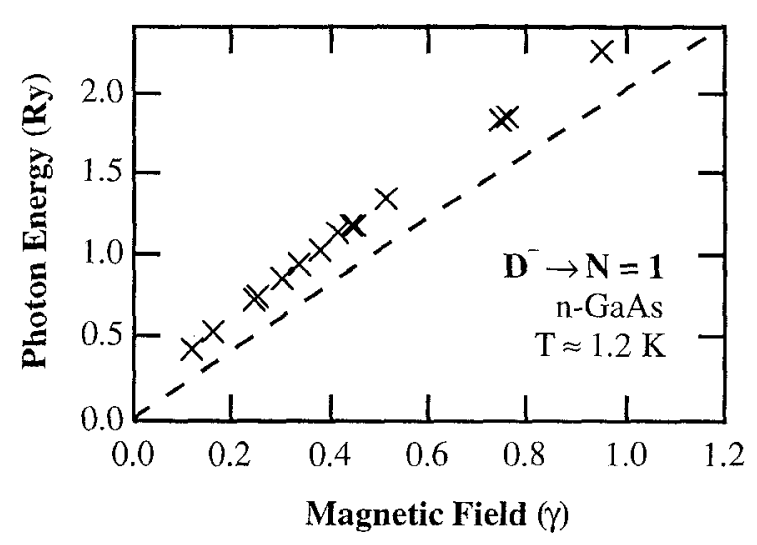

Fig. 2: Transitions from $\mathrm{D}^{-}$to $\mathrm{N}=1$ in $\mathrm{n}-\mathrm{GaAs}$. Units and dashed line are the same as in Fig. 1.

\section{C.3 Transitions to stable hydrogenic states}

The strongest features observed in the magneto-spectra are transitions from the lowest hydrogenic state to stable excited hydrogenic states. At zero field the hydrogenic states bear the familiar atomic labels $\mathrm{nlm}$, the ground state being denoted $1 \mathrm{~s}$. In high field notation, $\mathrm{Nmv}$, the quantum number $\mathrm{N}=0,1,2, \ldots, \infty$ represents the Landau level, $\mathrm{m}=\mathrm{N}, \mathrm{N}-1, \mathrm{~N}-2$, $\ldots .,-\infty$, the magnetic quantum number and $v=0,1,2, \ldots, \infty$, the number of nodes of the wavefunction in the magnetic field direction. In this notation the ground state is 000 .

The electric dipole selection rules are that the parities of the initial and final states are different, i.e. $\pi_{i} \pi_{f}=-1$, where $\pi$, the parity of a state, is given by $(-1)^{(m+\gamma)}$. The ground state is even parity and transitions are therefore only observed to odd-parity final states. Further, $\Delta \mathrm{m}= \pm 1$ for transitions for $\mathbf{k} \| \mathbf{B}$, the Faraday configuration, which was employed. (Here $\mathbf{k}$ is the wave-vector of the radiation.) Reflection of the radiation in the light pipe means that some component of $\mathbf{k} \perp \mathbf{B}$ falls on the sample (the Voigt configuration) allowing transitions with $\Delta \mathrm{m}=0$, e.g. $1 \mathrm{~s}_{0}$ to $2 \mathrm{p}_{0}$ to be observed. Data are presented in Fig. 3. 


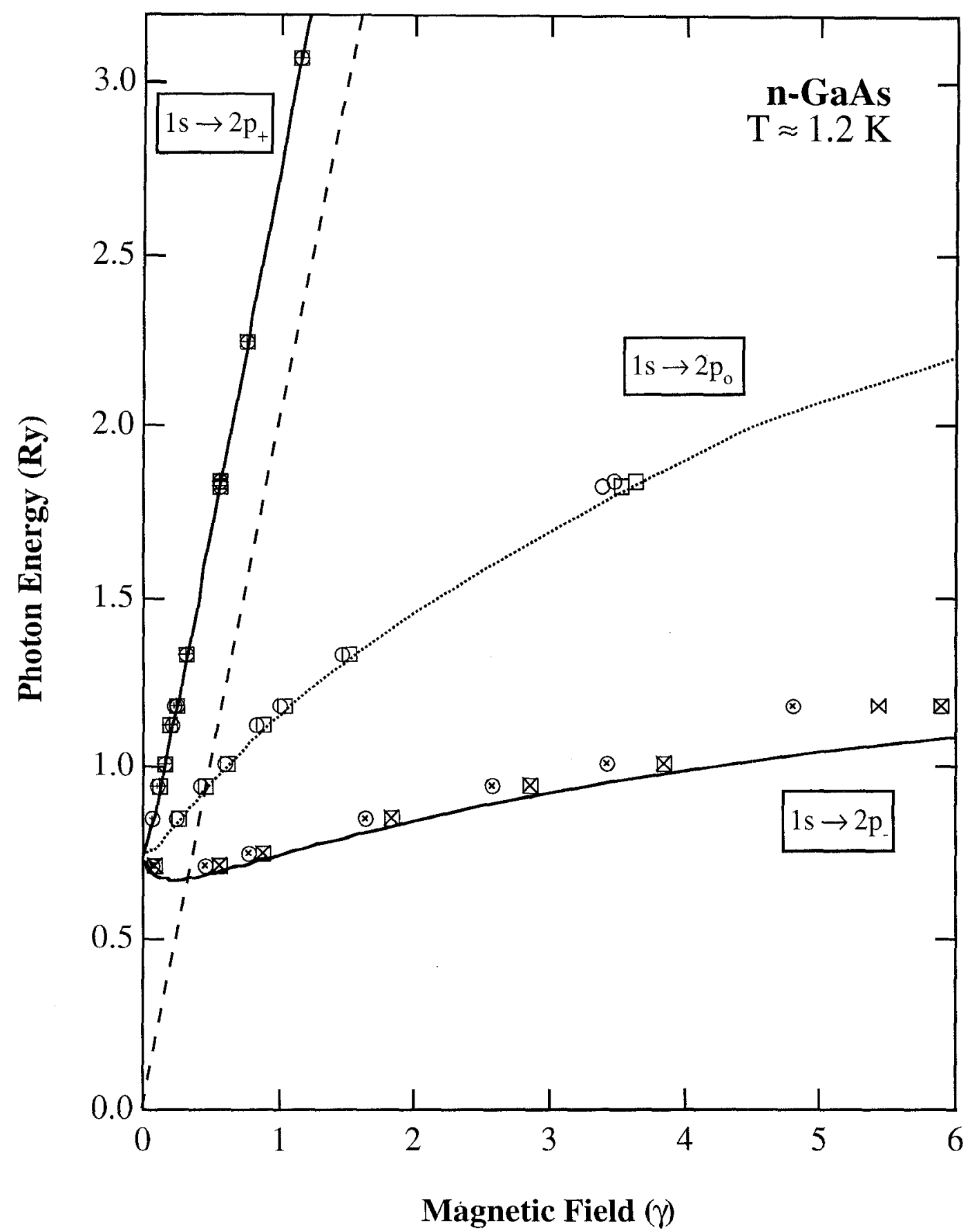

Fig. 3: Transitions between the ground and several higher hydrogenic states. The dashed line has the same meaning as in Fig. 1. The curves indicate the calculations of Makado and McGill [4] for the hydrogen atom. Full lines represent allowed transitions in the Faraday configuration, the dotted line a forbidden transition. In the case of the hydrogen atom, $R y=13.6 \mathrm{eV}$ and $\gamma=2.35 \times 10^{5} \mathrm{~T}$. The symbols indicate the present experimental data for $n$-GaAs. For this solid-state analogue of the hydrogen atom, Ry $=5.72 \mathrm{meV}$ and $\gamma=$ $6.57 \mathrm{~T}$. The two sets of data (three in the highest field case) at each laser energy correspond to different donors, believed to be $\mathrm{S}$ (circles), $\mathrm{Si}$ (squares) and $\mathrm{Sn} / \mathrm{Se}$ (bow-tie). The simple hydrogen theory fits the experimental data quite well, but there is a systematic divergence which increases with field. This chemical effect is analysed in detail elsewhere [5]. Previous work in $n$-GaAs has been restricted to $\gamma<2.5$. 


\section{C.4 Transitions to metastable hydrogenic} states

Every zero-field atomic state can be given a high-field label. The converse is not true: some high-field states have no atomic counterparts. These are known as metastable or auto-ionising states and are discussed in detail, in the context of n-GaAs, by van Klarenbosch et al. [6]. Transitions to some long-lived members of such states have been observed in the present experiments and data are given in Fig. 4. The dominant features at low magnetic field are labelled N10. Of this set, only 110 (i.e., $2 \mathrm{p}+$ ) is a stable state; transitions to this state have already been presented in Fig. 3. No calculations of the energies of these metastable states as a function of magnetic field are known; the lines in Fig. 4 only serve to guide the eye.

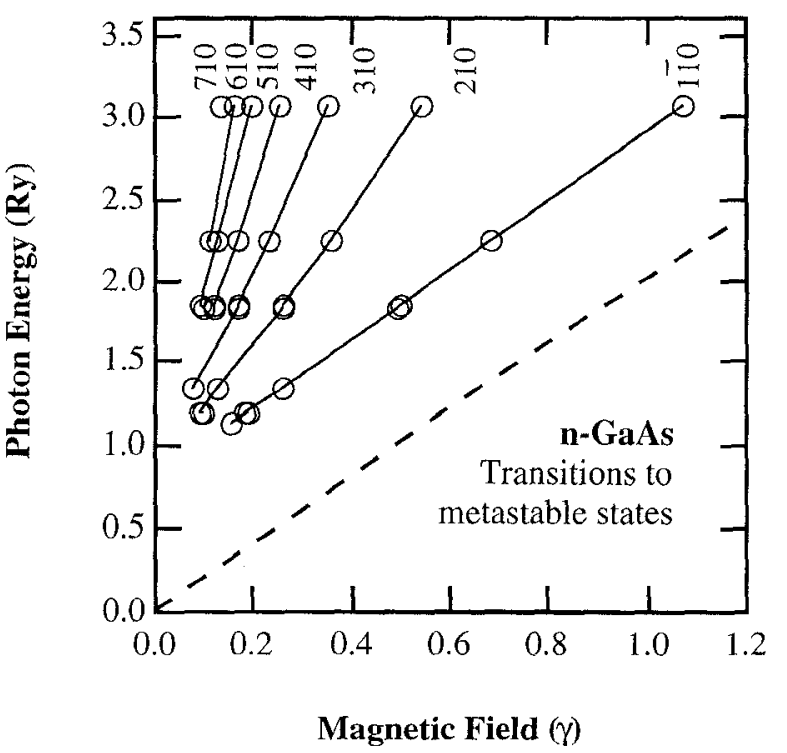

Fig. 4: Transitions to hydrogenic metastable states. The dashed line indicates the cyclotron resonance. The full lines serve only to guide the eye.

\section{Conclusion}

Far-infrared magnetospectroscopy is a powerful tool for investigating electronic states in semiconductor materials. The rich spectra for $n-G a A s$ show features attributed to transitions between Landau levels, transitions from $\mathrm{D}^{-}$bound states to Landau levels, and transitions from the hydrogenic ground state to hydrogenic higher states, both bound and metastable. The use of a ${ }^{3} \mathrm{He}$ system in a pulsed magnet has allowed simultaneous access to low temperatures and high fields not previously possible. Previous experimental work on these spectral features has been restricted to fields of about $6 \mathrm{~T}(\gamma \sim 1)$, with only one set of data extending as far as $15 \mathrm{~T}(\gamma \sim 2.3)$. The present work, to $42 \mathrm{~T}(\gamma \sim 6)$ therefore greatly extends the range of available data. It might be noted that $\gamma=6$ corresponds to a magnetic field for the hydrogen atom of $>10^{6} \mathrm{~T}$, a field not realisable in terrestrial experiments, but of interest in astrophysics.

\section{Acknowledgements}

This work was supported by the Australian Research Council, the University of Wollongong and the University of New South Wales. The sample was kindly provided by Prof. C. R. Stanley, Department of Electronics and Electrical Engineering, University of Glasgow.

\section{References}

[1] R. G. Clark et al., Physica B 201, 565 (1994).

[2] R. A. Lewis et al., in "High Magnetic Fields in Semiconductor Physics", edited by G. Landwehr (World Scientific, Singapore, in press).

[3] S. P. Najda et al., Semicond. Sci. Technol. 4, 439 (1989).

[4] P. C. Makado and N. C. McGill, J. Phys. C: Solid State Phys. 19, 873 (1986).

[5] R. J. Heron et al. (unpublished).

[6] A. van Klarenbosch et al., J. Appl. Phys. 67, 6323 (1990). 